\title{
PREFERÊNCIA POR ESTRATOS FLORESTAIS E POR SUBSTÂNCIAS ODORIFERAS EM ABELHAS EUGLOSSINAE (HYMENOPTERA, APIDAE)
}

\author{
Marcio Luiz de Oliveira ${ }^{1,2}$ \\ Lúcio Antônio de Oliveira Campos ${ }^{3}$
}

\begin{abstract}
STRATIFICATION AND SCENTS BAITS PREFERENCES IN EUGlossinae BEeS (HYMENOPTERA, APIDAE). Euglossinae bees of two areas of Terra Firme forest, near Manaus, Amazonas, Brazil were studied. During one year the collections were done fortnightly, using traps with eight kinds of scent baits. The traps were placed in the understory and in tree crowns. Some species showed a very clear vertical stratification in the forest. The comparison between the strata studied showed that the fauna of one understory is more similar to other than fauna of crowns and the similarity between fauna of understory and fauna of crown of the the two areaswas low. Some species were specialists while most were generalists in their choice of scent baits. Some species varied its preferences during the year.

KEY WORDS. Amazonia, Euglossinae, scent baits preferences, stratification
\end{abstract}

As primeiras observações sobre a relação de abelhas Euglossinae com orquídeas foram feitas por DARWIN (1862, apud BRAGA 1976); por CRUGER (1865) e por DUCKE (1901, 1902, apud MOURE 1969). Somente algum tempo depois esses estudos foram retomados por ALLEN $(1950,1954)$ e por PORSCH $(1955$, apud MOURE 1969). Estes pesquisadores pensavam que os machos visitavam as orquídeas para se alimentarem, dilacerando-lhes as pétalas. Entretanto, foram DoDSON \& FRYMIRE (1961) que notaram que os machos não dilaceravam as pétalas, mas raspavam-nas com as pernas dianteiras e retiravam substâncias que eram depositadas nas tíbias das pernas posteriores, no órgão tibial (DoDson et al. 1969).

A análise dos componentes dessas substâncias têm revelado que se tratam de odores comuns mas que possuem alguma atividade biológica ainda não suficientemente esclarecida (DoDSON et al. 1969). Esses odores podem conter muitos compostos tais como terpenóides, compostos aromáticos, compostos aminóides,

1) Departamento de Ciências da Natureza, Universidade Federal do Acre. 69915-900 Rio Branco, Acre, Brasil.

2) Correspondências: Departamento de Biologia, Faculdade de Filosofia Ciências e Letras, Universidade de São Paulo. 14040-901 Ribeirão Preto, São Paulo, Brasil.

E-mail: mlolivei@usp.br

3) Departamento de Biologia Geral, Universidade Federal de Viçosa. 36570-000 Viçosa, Minas Gerais, Brasil. 
hidrocarbonetos, e mais freqüentemente, monoterpenos (WILLIAMS \& WHITTEN 1983). Os compostos aromáticos e os terpenóides constituindo certos óleos voláteis ocorrem também em fontes não florais como fungos e madeiras podres que também são procuradas pelos machos (DRESSLER 1982; ACKERMAN 1985). Os machos visitam ainda outras famílias de plantas para coletar substâncias odoríferas como Araceae, Gesneriaceae, Solanaceae e Euphorbiaceae (WiLliams \& WhitTEN 1983).

DRESSLER (1982) cita que a princípio, pensava-se que o odor emitido pelas orquídeas imitava o feromônio das fêmeas de Euglossinae, o que atrairia os machos que por sua vez promoveriam a polinização. Este mesmo autor informa que têm sido sugerido que os machos utilizam essas substâncias para atrair outros machos para um mesmo local, afim de formarem sítios de acasalamento, ou mesmo que os machos incorporam e transformam essa substâncias em feromônios sexuais afim de atrair fêmeas para o acasalamento.

Foi a partir dessas descobertas, e com a utilização no campo de substâncias análogas às produzidas pelas orquídeas, que as coletas e conseqüentemente $o$ conhecimento da biologia dos Euglossinae aumentou.

Entretanto, nos trabalhos realizados até o presente, não se procurou comparar a composição faunística das copas em relação ao sub-bosque, assumindo-se que os machos de Euglossinae seriam atraídos para as substâncias odoríferas, independentemente das alturas em que estas sejam colocadas.

Os objetivos deste trabalho foram: conhecer a fauna de abelhas Euglissinae das copas e dos sub-bosques das florestas contínuas de terra firme da Amazônia Central e ainda suas preferências por substâncias odoríferas.

\section{MATERIAL E MÉTODOS}

A localização e a descrição da área de estudos, bem como as informações sobre a metodologia de coleta encontram-se em OLIVEIRA \& CAMPOS (1995)

Os valores de diversidade da fauna de Euglossinae para as duas alturas, foram calculados pelo índice H' de Shannon (MagurRaN 1988). Este índice leva em consideração o número de espécies (riqueza) e a distribuição dos indivíduos entre as espécies (equitabilidade) (PEET 1974). Os valores obtidos por este índice foram comparados por uma variação do teste "t", proposta por HUTCHESON (1970). Os valores de equitabilidade para as duas alturas foram calculados pelo índice J' de Pielou (LUDWIG \& REYNOLDS 1988).

A similaridade faunística entre as duas alturas foi obtida pelo coeficiente de dissimilaridade de distância da corda (PIELOU 1984; LudWIG \& REYNOLDS 1988). Este coeficiente mede a dissimilaridade entre duas amostras, portanto, quanto maior o valor obtido, menor a semelhança entre elas. Com os valores obtidos foram montados dendrogramas de dissimilaridade de acordo com o método de agrupamento UPGMA (Unweighted pair-grouping method analysis) (LUDWIG \& REYNOLDS 1988). 


\section{RESULTADOS}

De um modo geral, a maioria das espécies ocorreu nos dois estratos (Tab. I). Espécies que foram pouco comuns como Euglossa laevecincta, E. prasina, E. viridis e Euglossa sp. 1 foram capturadas exclusivamente junto as copas, enquanto $E$. piliventris, Euglossa sp: 3, Euplusia vidua e Exaerete smaragdina foram capturadas exclusivamente nos sub-bosques. Espécies mais abundantes como E. augaspis e $E$. crassipunctata foram muito mais comuns nas copas, enquanto $E$. chalybeata foi muito mais comum no sub-bosque (Fig. 1).

Tabela I. Abelhas Euglossinae coletadas nas copas e nos sub-bosques de duas áreas de mata contínua de terra firme na Amazônia Central.

\begin{tabular}{|c|c|c|c|c|c|c|c|}
\hline \multirow{2}{*}{ Espécies } & \multicolumn{2}{|c|}{ Área 1401} & \multicolumn{2}{|c|}{ Área 1501} & \multirow{2}{*}{$\begin{array}{l}\text { Total } \\
\text { copa }\end{array}$} & \multirow{2}{*}{$\begin{array}{c}\text { Total } \\
\text { sub-bosque }\end{array}$} & \multirow{2}{*}{ Total } \\
\hline & Copa & Sub-bosque & Copa & Sub-bosque & & & \\
\hline Euglossa analis Westwood, 1840 & 1 & 2 & 1 & 1 & 2 & 3 & 5 \\
\hline E. augaspis Dressler, 1982 & 113 & 40 & 113 & 30 & 226 & 70 & 296 \\
\hline E. avicula Dressler, 1982 & 81 & 55 & 86 & 57 & 167 & 112 & 279 \\
\hline E. bidentata Dressler, 1982 & - & - & 2 & 1 & 2 & 1 & 3 \\
\hline E. chalybeata Friese, 1925 & 38 & 83 & 71 & 160 & 109 & 243 & 352 \\
\hline E. cognata Moure, 1970 & 2 & - & 12 & 2 & 14 & 2 & 16 \\
\hline E. crassipunctata Moure, 1968 & 50 & 14 & 72 & 15 & 122 & 29 & 151 \\
\hline E. decorata Smith, 1854 & 6 & 8 & 3 & 1 & 9 & 9 & 18 \\
\hline E. gaianii Dressler, 1982 & 7 & 2 & 4 & 3 & 11 & 5 & 16 \\
\hline E. ignita Smith, 1854 & 8 & 6 & 7 & 3 & 15 & 9 & 24 \\
\hline E. imperialis Cockerell, 1922 & 1 & 1 & 5 & 5 & 6 & 6 & 12 \\
\hline E. intersecta Latreille, 1938 & - & 2 & 4 & 4 & 4 & 6 & 10 \\
\hline E. ioprosopa Dressler, 1982 & 3 & 1 & 5 & - & 8 & 1 & 9 \\
\hline E. iopyrma Dressler, 1982 & 2 & 1 & 6 & 3 & 8 & 4 & 12 \\
\hline E. laevicincta Dressler, 1982 & - & - & 1 & - & 1 & - & 1 \\
\hline E. mixta Friese, 1989 & 11 & 16 & 26 & 14 & 37 & 30 & 67 \\
\hline E. modestior Dressler, 1982 & 5 & 2 & - & 2 & 5 & 4 & 9 \\
\hline E. mourei Dressler, 1982 & 17 & 6 & 15 & 4 & 32 & 10 & 42 \\
\hline E. parvula Dressler, 1982 & 8 & 4 & 16 & 4 & 24 & 8 & 32 \\
\hline E. piliventris Guérin, 1845 & - & - & - & 2 & - & 2 & 2 \\
\hline E. prasina Dressler, 1982 & - & - & 2 & - & 2 & - & 2 \\
\hline E. retroviridis Dressler, 1982 & 7 & 2 & 9 & 1 & 16 & 3 & 19 \\
\hline E. stilbonota Dressler, 1982 & 137 & 174 & 217 & 257 & 354 & 431 & 785 \\
\hline E. viridifrons Dressler, 1982 & 6 & 2 & 6 & 2 & 12 & 4 & 16 \\
\hline E. viridis (Perty, 1833) & 2 & - & 3 & - & 5 & - & 5 \\
\hline Euglossa sp. 1 & - & - & 3 & - & 3 & - & 3 \\
\hline Euglossa sp. 2 & 1 & 1 & - & - & 1 & 1 & 2 \\
\hline Euglossa sp. 3 & - & - & - & 1 & - & 1 & 1 \\
\hline Eulaema bombiformis (Packard, 1869) & 3 & 3 & 4 & 3 & 7 & 6 & 13 \\
\hline E. meriana (Olivier, 1789) & 14 & 28 & 14 & 24 & 28 & 52 & 80 \\
\hline E. cingulata (Fabricius, 1804) & 1 & 4 & - & 1 & 1 & 5 & 6 \\
\hline E. mocsaryi (Friese, 1899) & 8 & 9 & 6 & 5 & 14 & 14 & 28 \\
\hline Eufriesea pulchra Smith, 1854 & 2 & - & - & 1 & 2 & 1 & 3 \\
\hline Euplusia ornata (Mocsáry, 1896) & 4 & 1 & 2 & 2 & 6 & 3 & 9 \\
\hline E. vidua Moure, 1976 & - & 1 & - & - & 1 & - & 1 \\
\hline Exaerete frontalis (Guérin, 1845) & 15 & 35 & 7 & 23 & 22 & 58 & 80 \\
\hline E. smaragdina (Guérin, 1845) & - & 5 & - & 5 & - & 10 & 10 \\
\hline E. trochantherica (Friese, 1900) & - & 1 & 1 & 1 & 1 & 2 & 3 \\
\hline Total de individuos & 553 & 508 & 724 & 637 & 1277 & 1145 & 2422 \\
\hline Total de espécies & 28 & 29 & 30 & 31 & 35 & 33 & 38 \\
\hline
\end{tabular}




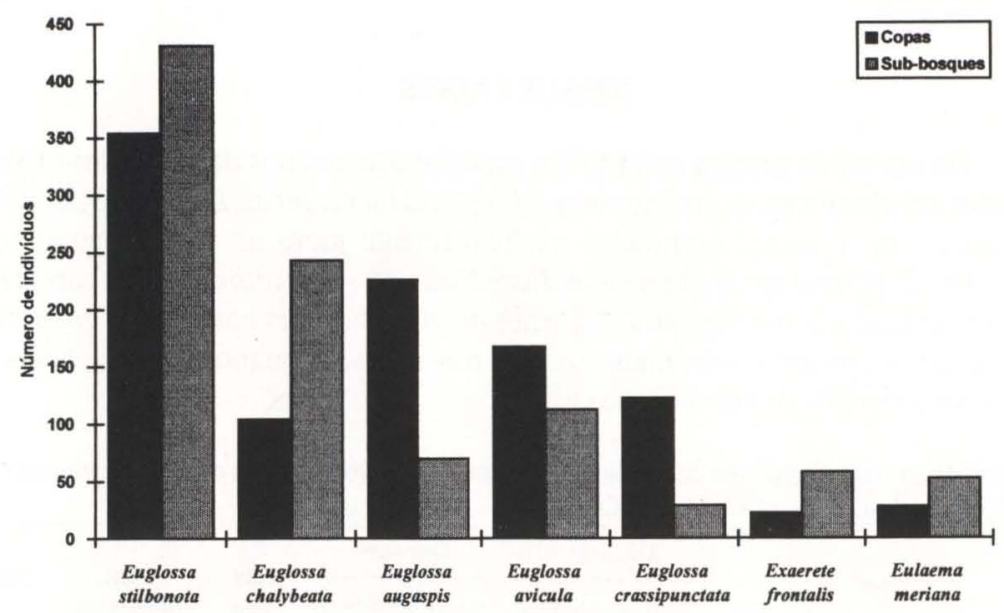

Fig. 1. Abelhas Euglossinae mais abundantes nas copas e nos sub-bosques de duas áreas de mata contínua de terra firme na Amazônia Central.

O número de indivíduos de cada espécie variou entre os estratos, mas não foi grande a diferença entre o total coletado nas copas e o coletado nos sub-bosques $(\mathrm{X} 2=0,235 ; \mathrm{G} . \mathrm{L} .=1 ; \mathrm{p}=0,628)$. Por sua vez, o número de espécies e o total de espécies foi praticamente o mesmo nos dois estratos (Tab. I). A diversidade encontrada nas copas foi superior à dos sub-bosques (Tab. II).

Tabela II. Indice de diversidade de Shannon ( $\left.\mathrm{H}^{\prime}\right)$ e de equitabilidade de Pielou (J') para as copas e sub-bosques.

\begin{tabular}{lcc}
\hline \multicolumn{1}{c}{ Estrato } & $\mathrm{H}^{\prime}$ & $\mathrm{J}^{\prime}$ \\
\hline Copas & 2,346 & 0,660 \\
Sub-bosques & 2,123 & 0,607 \\
\hline
\end{tabular}

*. Todos os valores de diversidade foram significativamente diferentes $(t=4,419, G$. L. $=2206$, $p=5,2 \times 10$ e -6$)$.

Ao se comparar a fauna de Euglossinae das copas e dos sub-bosques das duas áreas de mata estudadas, houve maior similaridade entre os sub-bosques, do que entre as copas, ou mesmo do que entre as copas e os sub-bosques (Tab. III). Pelo dendrograma de dissimilaridade, verifica-se que os sub-bosques ficaram localizados em um agrupamento e as copas em outro (Fig. 2).

$\mathrm{Na}$ área-1401, a isca que atraiu maior número de indivíduos foi cineol seguido de vanilina, mas foi salicilato de metila que atraiu o maior número de espécies. Semelhantemente, na área 1501, cineol e vanilina foram as iscas que mais atrairam indivíduos, sendo que o primeiro atraiu também o maior número de espécies. Em termos gerais, muito embora cineol tenha atraído o maior número de indivíduos (1097), foi salicilato de metila que atraiu o maior número de espécies (20) (Tab. IV).

Algumas espécies como E. cognata e E. viridis foram atraídas exclusivamente para salicilato de metila, enquanto Euglossa sp. 2 foi atraída somente para 
cineol. E. augaspis, E. avicula, E. crassipuncatata, E. decorata e E. mourei, apesar de comuns, foram atraídas quase que exclusivamente por vanilina. E. chalybeata $\mathrm{e}$ E. stilbonota o foram quase que exclusivamente por cineol, enquanto E. mixta o foi por salicilato de metila.

Tabela III. Dissimilaridade entre a fauna de abelhas Euglossinae nas copas e nos sub-bosques das duas áreas estudadas, organizadas de modo crescente e calculada pelo índice de distância da corda *.

\section{Ambientes}

Coeficiente de dissimilaridade *

\begin{tabular}{ll}
\hline Sub-bosque $1401 \times$ sub-bosque 1501 & 0,21 \\
Copa $1401 \times$ copa 1501 & 0,23 \\
Sub-bosque $1401 \times$ copa 1501 & 0,37 \\
Sub-bosque $1501 \times$ copa 1501 & 0,48 \\
Sub-bosque $1401 \times$ copa 1401 & 0,51 \\
Sub-bosque $1501 \times$ copa 1401 & 0,65
\end{tabular}

*. Indice varia de 0 a 1,414 .

Tabela IV. Total de abelhas Euglossinae coletadas com substâncias odoriferas em duas áreas de mata continua de terra firme na Amazônia Central. (A) Acetato de benzila, (B) benzoato de benzila, (C) cineol, (E) eugenol, (K) escatol, (M) cinamato de metila, (S) salicilato de metila, (V) vanilina.

\begin{tabular}{llrrrrrrrrr}
\hline & C & E & K & A & M & S & B & V & Total \\
\hline Área 1401 & Total de indivíduos & 453 & 49 & 24 & 32 & 45 & 104 & 2 & 352 & 1061 \\
& Total de espécies & 18 & 7 & 8 & 8 & 8 & 20 & 2 & 14 & 32 \\
Área 1501 & Total de indivíduos & 644 & 48 & 34 & 66 & 44 & 144 & 8 & 373 & 1361 \\
& Total de espécies & 18 & 12 & 12 & 14 & 6 & 16 & 4 & 15 & 36 \\
\hline
\end{tabular}

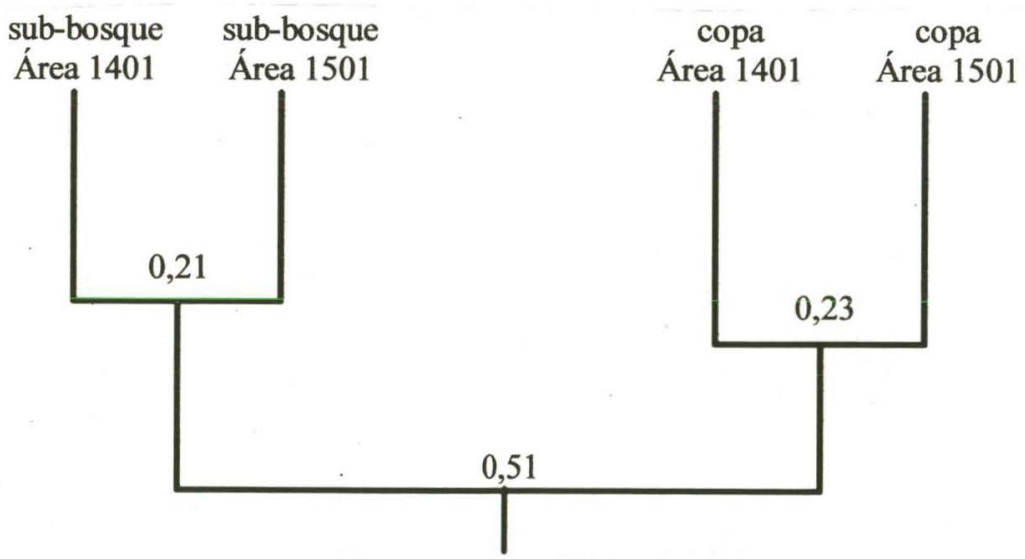

Fig. 2. Dendrograma de dissimilaridade da fauna de abelhas Euglossinae em relação as copas e aos sub-bosques de duas áreas de matas contínuas de terra firme na Amazônia Central, obtido pelo indice de dissimilaridade de distância da corda e pelo método de agrupamento UPGMA. 
A três espécies mais abundantes variaram suas preferências por substâncias odoríferas, ao longo do ano, nas duas áreas (Fig. 3). No mês de junho, E. stilbonota foi mais rara na àrea 1401 e dividiu sua preferência entre cineol e outras substâncias como salicilato de metila e vanilina; na área 1501, dividiu sua preferência entre cineol, eugenol e vanilina. Já, E. chalybeata que de início preferia basicamente cineol, dividiu sua preferência com várias outras substâncias a partir de dezembro. Algo semelhante aconteceu com E. augaspis que dividiu sua preferência entre vanilina e outras substâncias a partir de novembro.
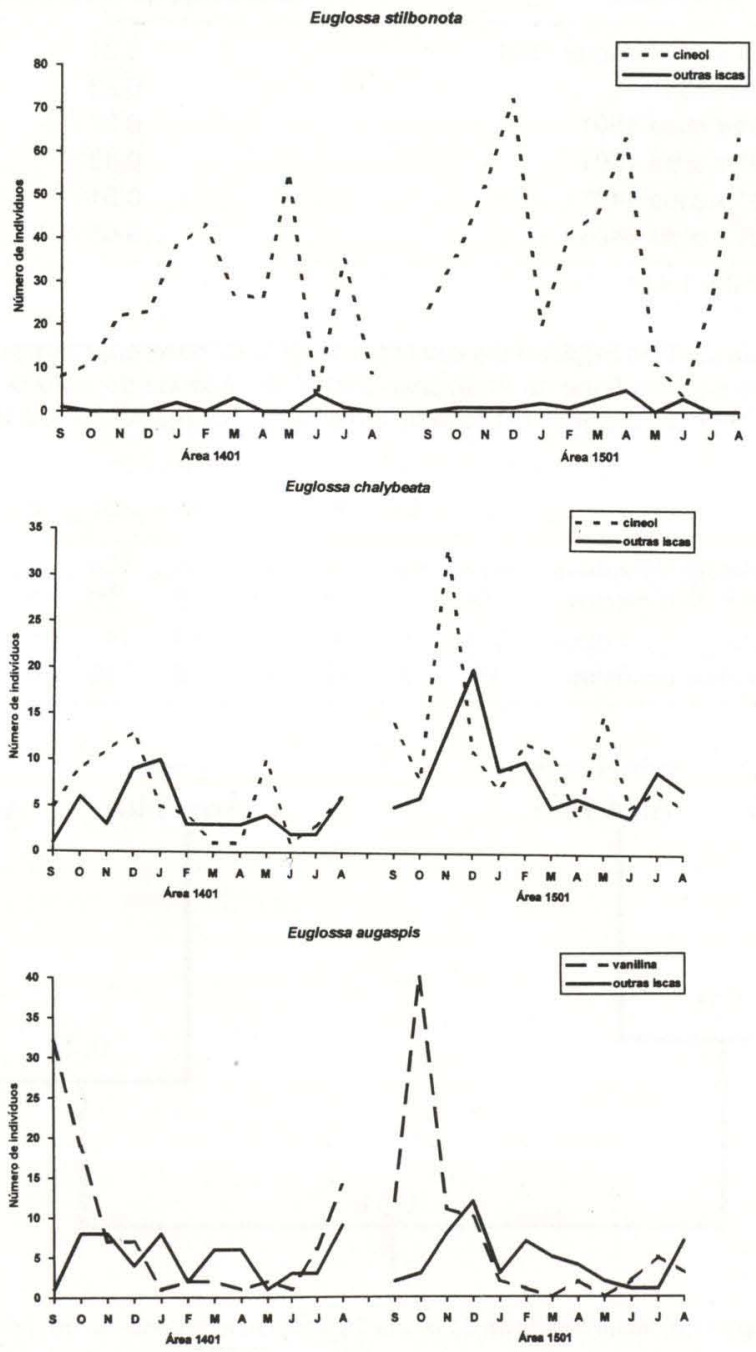

Fig. 3. Variação nas preferências por iscas de cheiro das três espécies de Euglossinae mais abundantes em duas áreas de mata contínua de terra firme na Amazônia Central. 


\section{DISCUSSÃO}

Apesar da altura entre as armadilhas com substâncias odoríferas do sub-bosque e da copa ser relativamente pequena, algo em torno de 12 metros, ela foi suficiente para verificar as preferências de algumas espécies por algum destes dois ambientes. É possível que armadilhas situadas nas copas a alturas superiores a 30 metros, que é a média de altura das copas nessas florestas (BIERREGAARD \& LOVEJOY 1988), ou mesmo em diferentes alturas, nos mostre uma estratificação mais bem definida.

Entretanto, V.E. Shelford (apud DowDY 1951) assinala que a migração de animais de um estrato para outro torna os limites de divisão entre os estratos difíceis de se delinear em alguns casos, mas admite que o reconhecimento do estrato é essencial e mais importante que uma definição rígida do mesmo.

As copas apresentaram maior riqueza, abundância e também maior diversidade, entretanto isso varia de grupo para grupo. DowDY (1951) por exemplo, coletou artrópodos em solos, folhiço $(5 \mathrm{~cm})$, ervas $(60 \mathrm{~cm})$, arbustos $(1,37 \mathrm{~m})$ e árvores $(2,90 \mathrm{~m})$ de florestas de carvalho e cedro. Verificou que a abundância relativa foi maior no folhiço, enquanto a riqueza foi maior nas ervas. Concluiu que variação na temperatura e outros fatores físicos e biológicos provavelmente formam a base das estratificações e que alimentos e locais para nidificação talvez expliquem os picos das populações nos estratos, nas florestas temperadas.

ERWIN (1982) estima que a fauna de artrópodos nas copas deva ser pelo menos duas vezes mais rica que a do solo, em florestas tropicais. SUTTON et al. (1983) encontraram em muitos casos, uma clara estratificação e marcada preferência de alguns grupos de insetos, inclusive Hymenoptera, pelas copas em florestas tropicais do Panamá, Papua-Nova Guiné e Brunei. Na maioria dos casos, as alturas preferidas estavam em torno de 20 e 30 metros.

WOLDA \& ROUBIK (1986) verificaram que espécies de abelhas do gêneros Rhinetula e Ptiloglossa que nidificam no solo, foram capturadas principalmente por armadilhas situadas a três metros de altura. Megalopta que nidifica em madeira foi mais abundante a 27 metros de altura. Estes autores também concordam com a influência de fatores como alimentação e locais de nidificação, mas no sabem quais desses fatores é predominante.

COLLINS \& JENNINGS (1987) não encontraram preferência por altura de nidificação em três espécies de Eumenidae (Hymenoptera) que estudaram, provavelmente por terem instalados os ninhos-armadilha para captura a alturas de no máximo 1,5 metros. FRANKIE et al. (1988) colocaram ninhos para capturas a 0,5 e 2,5 metros de altura, para estudar preferências de nificação de abelhas do gênero Centris e verificaram que uma grande percentagem fundou ninhos a 2,5 metros. MORATO (1993) colocou armadilhas nas árvores a 1,5; oito e quinze metros de altura, para verificar preferências de nidificação de abelhas e vespas solitárias nas mesmas florestas em que foi realizado o presente estudo e verificou que a maioria de vespas e abelhas fundou ninhos a oito e quinze metros.

É muito difícil explicar a razão de tais preferências. SALMAH et al. (1990), assinalam que nas florestas primárias as condições microclimáticas variam entre $o$ interior do sub-bosque e as margens das copas. Os sub-bosques são caracterizados 
por menores temperaturas, menor intensidade luminosa, fracas correntes de ar, alta umidade e pequenas flutuações nessas variáveis, enquanto nas margens das copas ocorre o inverso e com flutuações mais amplas nessas variáveis. Estes autores consideram ainda que esses gradientes microclimáticos produziriam uma série de microhabitats nos quais diferentes animais, inclusive abelhas da família Apidae, se adaptariam diferencialmente.

É possível que a estratificação da fauna de Euglossinae esteja relacionada com a estratificação das Orchidaceae, isto sem desprezar os demais fatores citados anteriormente, já que é principalmente nestas plantas que os machos retiram substâncias odoríferas. BRAGA (1987), por exemplo, cita que essas plantas estão situadas em diferentes gradientes verticais no interior dos ecossistemas vegetacionais.

A maior semelhança entre fauna de Euglossinae, primeiramente dos sub-bosques e depois das copas das duas áreas, ao invés de sub-bosque e copa de uma mesma área mostra que a maioria das espécies apresenta preferência por um destes estratos e que esta preferência é concordante nas duas áreas.

Dos trabalhos realizados nas florestas de terra firme da Amazônia Central até este momento, este foi o que utilizou maior número de substâncias odoríferas e incluiu as que foram utilizadas pelos outros autores. As oito substâncias atraíram 38 espécies, entretanto, verificou-se que bastavam cinco delas (cineol, eugenol, escatol, salicilato de metila e vanilina), para se obter o mesmo resultado (Tab. IV).

As substâncias mais atrativas foram cineol, vanilina e salicilato de metila. Nos trabalhos de BRAGA (1976), BECKER et al. (1991) e MorATO et al. (1992), cineol atraiu o maior número de indivíduos e de espécies. No de POWELL \& POWELL (1987), cineol também atraiu maior número de indivíduos, mas foi salicilato de metila que atraiu maior número de espécies. Este resultado é semelhante ao obtido aqui para a área 1401. As pequenas diferenças entre estes trabalhos, apesar de usarem quase sempre as mesmas substâncias, talvez possa ser explicado pelo fato de terem sido realizados em épocas, ambientes e com métodos de coleta diferentes. Por outro lado, vários autores têm verificado que, apesar de empregarem muitas outras substâncias odoríferas, o padrão de preferência tem se repetido. Como exemplo, a atração de E. stilbonota quase que exclusivamente por cineol e de $E$. chalybeata e E. augaspis por várias iscas já havia sido verificada pelos autores citados anteriormente.

Os poucos indivíduos de E. stilbonota que apareceram em junho, um dos meses mais secos do ano com apenas $123,3 \mathrm{~mm}$ de precipitação, dividiram suas preferências entre cineol, salicilato de metila e vanilina. É possível que diante de uma situação tão extrema, os recursos naturais tenham se tornado tão escassos que os poucos machos que estiveram ativos nesse período se viram forçados a obter substâncias odoríferas em todas as fontes disponíveis, mesmo naquelas menos costumeiras.

Euglossa chalybeata e E. augaspis variaram suas preferências por substâncias odoríferas durante grande parte do ano. Ao que tudo indica, estas duas espécies são generalistas em relação às fontes naturais de substâncias odoríferas que visitam e seriam atraídas para as que estivessem mais disponíveis ao longo do ano. 
Esta variação sazonal nas preferências já tinha sido observada por PEARSON \& DRESSler (1985), ACKERMAN (1989), WitTMANN et al. (1989) e REBElo \& GARófAlo (1991). Para ZimMERMAN \& MADRINAN (1988) e ACKERMAN (1989), alguma variação nas preferências pode ser explicada, pelo menos parcialmente, por mudanças na estrutura etária da população, sendo que os primeiros autores verificaram que machos jovens visitam principalmente substâncias odoríferas, ao passo que machos velhos visitam principalmente fontes de néctar, e que os machos jovens necessitariam coletar essas substâncias antes de estabelecerem seus territórios. Do mesmo modo, REBELO \& GARÓFALO (1991) verificaram que 77\% dos machos que coletavam em substâncias odoríferas eram jovens. WiTTMANN et al. (1989) citam que além deste fator, as modificações ambientais podem influir sobre o metabolismo da glândula mandibular, supostamente responsável pela secreção dos compostos que são derivados das substâncias coletadas pelos Euglossinae na natureza. A variação sazonal na preferência por substâncias odoríferas pode estar ainda relacionada com o padrão de floração das orquídeas, principal fonte de obtenção de substâncias odoríferas pelos Euglossinae.

WILLIAMS \& WHITTEN (1983) assinalam contudo que, dados sobre a atratividade de machos de Euglossinae para determinados compostos precisam ser vistos com precaução. Isto porque, segundo estes autores, alguns destes compostos tidos como pouco atrativos, têm sido usados poucas vezes em trabalhos de campo e que por outro lado, a pureza química e a composição isomérica de outros ainda são desconhecidos.

Algumas substâncias odoríferas, como é o caso de salicilato de metila, até agora só foram encontradas em três espécies de orquídeas, Cycnoches sp., Stanhopea candida e S. panamensis (Williams \& WHITTEN 1983). Neste trabalho verificou-se que esta foi a substância que atraiu maior número de espécies de Euglossinae e, se isto reflete a realidade, quaisquer perturbações do ambiente natural que afetem essas poucas espécies de orquídeas, poderão afetar as diversas espécies de abelhas que as visitam.

AGRADECIMENTOS. Ao Prof. Dr. Pe. Jesus S. Moure (Departamento de Zoologia, Universidade Federal do Paraná) pelo auxílio na identificação das espécies. A Elder F. Morato e Paulo de Marco Jr. pelas criticas e sugestões. Ao PDBFF pelo financiamento, esta é a publicação número 162 deste projeto.

\section{REFERÊNCIAS BIBLIOGRÁFICAS}

ACKERMAN, J.D. 1985. Euglossine bees and their nectar hosts, p. 225-233. In: W.G. D'ARCY \& M.D. CORREA (Eds). The botany and natural history of Panama: La botanica e historia natural de Panama. St. Louis, Missouri Botanical Garden.

1989. Geographic and seasonal variation in fragrance choices and preferences of male euglossine bees. Biotropica 21 (4): 340-347.

BECKER, P.; J.S. Moure \& F.J.A. PERALTA. 1991. More about euglossine bees in amazonian forest fragments. Biotropica 23 (4b): 586-591. 
BIERREgaARD JR., R.O. \& T.E. Lovejoy. 1988. Birds in Amazonian forest fragments: effects of insularization, p. 1564-1579. In: H. QUELLET (Ed.). Acta XIX Cong. Int. Ornith., Otawa, University of Otawa Press, vol. 2, 1409p.

BRAGA, P.I.S. 1976. Atração de abelhas polinizadoras de Orchidaceae com auxílio de iscas-odores na campina, campinarana e floresta tropical úmida da região de Manaus. Ciência e Cultura 28 (7): 767-773. 1987. Orquídeas, Biologia floral. Ciência Hoje 5 (28): 53-55.

Collins, J.A. \& D.T. JeNNINGS. 1987. Nesting height preferences of eumenid wasps (Hymenoptera, Eumenidae) that prey on spruce budworm (Lepidoptera, Tortricidae). Ann. Entomol. Soc. Amer. 80: 435-438.

Dodson, C.H. \& G.P. FrYMIRE. 1961. Natural pollination of orchids. Miss. Bot. Gard. Bull. 49 (9): 133-152.

Dodson, C.H.; R.L. DressleR; H.G. Hills; R.M. Adams \& N.H. Williams. 1969. Biologically active compounds in orchid fragrances. Science 164 (13): 1243-1249.

DowDY, W.W. 1951. Further ecological studies on stratification of the Arthropoda. Ecology 32 (1): 37-52.

DRESSLER, R.L. 1982. Biology of the orchid bees (Euglossini). Ann Rev. Ecol. Syst. 13: 373-394.

ERWIN, T.L. 1982. Tropical forest: Their richness in Coleoptera and other arthrpods species. The Coleopterists Bulletin 36 (1): 74-75.

Frankie, G.W.; S.B. Vinson \& J.F. BARTHELl. 1988. Nest site and habitat preferences of Centris bees in the Costa Rican dry forest. Biotropica 20 (4): 301-310.

HutCheson, K. 1970. A test for comparing diversities based on the Shannon formula. J. Theor. Biol. 29: 151-154.

LUDWIG, J.A. \& J.F. REYNOLDS. 1988. Statistical ecology: A primer on methods and computing. New York, John Wiley \& Sons, 337p.

MAGURRAN, A.E. 1988. Ecological diversity and its measurement. Princeton, Princeton University Press, 179p.

MORATO, E.F. 1993. Efeitos da fragmentação florestal sobre vespas e abelhas solitárias em uma área da Amazônia Central. Dissertação de Mestrado, não publicada, Uiversidade Federal de Viçosa, Viçosa, 105p.

Morato, E.F.; L.A.O. CAMPos \& J.S. Moure. 1992. Abelhas Euglossini (Hymenoptera, Apidae) coletadas na Amazônia Central. Revta bras. Ent. 36 (4): 767-771.

Moure, J.S. 1969. Abelhas euglossinas e orquídeas. Ciência e Cultura 21 (2): 467-468.

OliveirA, M.L. \& L.A.O. CAMPOS. 1995. Abundância, riqueza e diverisdade de abelhas Euglossinae (Hymenoptera, Apidae) em florestas contínuas de terra firme na Amazônia Central, Brasil. Revta bras. Zool. 12 (3): 547-556.

Pearson, D. L. \& R. L. Dressler. 1985. Two-year study of male orchid bee (Hymenoptera, Apidae, Euglossini) attraction to chemical baits in lowland south-eastern Peru. Jour. Trop. Ecol. 1: 37-54. 
PEeT, R.K. 1974. The measurement of species diversity. Ann. Rev. Ecol. Syst. 51 (3): 445-454.

PIELOU, E.C. 1984. The interpretation of ecological data. New York, John Wiley \& Sons, 263p.

Powell, A.H. \& G.N.V. PowelL. 1987. Population dynamics of male euglossine bees in amazonian forest fragments. Biotropica 19 (2): 176-179.

REBELO, J.M.M. \& C.A. GARófalo. 1991. Diversidade e sazonalidade de machos de Euglossini (Hymenoptera, Apidae) e preferência por iscas odores em um fragmento de floresta no sudeste do Brasil. Rev. Brasil. Biol. 51 (4): 787-799.

SALMAH, S.; T. INOUE, \& S.F. SAKAGAMI. 1990. An analysis of apid bee richness (Apidae) in Central Sumatra, p.139-174. In: S.F. SAKAGAMI; R. OHGUSHI \& D.W. RoubIK (Eds). Natural history of social wasps and bees in Equatorial Sumatra. Sapporo, Hokkaido University Press, 274p.

SutTON, S.L.; C.P. ASH \& A. GRUNDY. 1983. The vertical distribution of flying insects in lowland rain-forest of Panama, Papua, New Guinea and Brunei. Zool. Jour. Linn. Soc. 78: 287-297.

WiLliams, N.H. \& W.M. WhitTEN. 1983. Orchid floral fragrances and male euglossine bees: Methods and advances in the last sesquidecade. Biol. Bull. 164: 355-395.

Wittmann, D.; R. Radtke; M. Hoffmann \& B. Blochtein. 1989. Seasonality and seasonal changes in preferences for scent baits in Euplusia violacea in Rio Grande do Sul/Brazil (Hymenoptera, Apidae, Euglossini). Entomol. Gener. 14 (3/4): 217-221.

WolDA, H. \& D.W. RouBIK. 1986. Nocturnal bee abundance and seasonal bee activity in a panamanian forest. Ecology 67 (2): 426-433.

Zimmerman, J.K. \& S. R. MAdrinan. 1988. Age structure of male Euglossa imperialis (Hymenoptera, Apidae, Euglossini) at nectar and chemical sources in Panama. Jour. Trop. Ecol. 4: 303-306.

Recebido em 08.XII.1995; aceito em 27.XII.1996. 